\title{
Hybrid Method for Computing the Nearest Singular Polynomials
}

\author{
Lihong Zhi, Matu-Tarow Noda, Hiroshi Kai \\ Department of Computer Science, Ehime University, Japan \\ \{lzhi,noda,kai\}@hpc.cs.ehime-u.ac.jp \\ Wenda $\mathrm{Wu}$ \\ Beijing Municipal Computing Center, China \\ wdwu@mmrc.iss.ac.cn
}

\begin{abstract}
In this paper, we propose a combined symbolic-numeric algorithm for computing the nearest singular polynomial and its multiple zero. Explicit expressions of the minimal perturbation and the nearest singular polynomials are presented. A theoretical error bound and several numerical examples are given.
\end{abstract}

Keywords: Quadratic form; Minimization; Multiple roots. 


\section{Introduction}

An approach based on minimization of quadratic forms to study problems related to polynomials with inexact coefficients has been proposed by Karmarkar and Lakshman [4] [5]. In an earlier paper [8], we have applied it to the following problem. Given a monic polynomial $f$

$$
f=x^{m}+\sum_{j=1}^{m} f_{j} x^{m-j}, \quad f_{j} \in \mathbb{C},
$$

find a monic polynomial $h(x)$ with a multiple root

$$
h=(x-c)^{k}\left(x^{m-k}+\sum_{j=1}^{m-k} \phi_{j} x^{m-k-j}\right), \quad \phi_{j} \in \mathbb{C},
$$

such that

$$
\mathcal{N}^{(k)}:=\|f-h\|^{2}
$$

is minimized. Here $\mathbb{C}$ denotes the complex number field, and $\|f-h\|$ is the $l^{2}$-norm of its coefficient vector. Here and after, $c$ denotes unknown multiple root.

Let $\mathbf{A}$ be an $m \times(m-k)$ matrix with entries $a_{i j}=\left(\begin{array}{c}k \\ i-j\end{array}\right)(-c)^{i-j}$, where $\left(\begin{array}{c}p \\ q\end{array}\right)=0$ if $q>p$ or $q<0$. We define

$$
\begin{aligned}
\mathbf{u} & :=\left[f_{1}, f_{2}, \ldots, f_{m}\right]^{T}, \\
\mathbf{v} & :=\left[\phi_{1}, \phi_{2}, \ldots, \phi_{m-k}\right]^{T}, \\
\mathbf{w} & :=\left[a_{10}, \cdots, a_{k 0}, 0, \cdots, 0\right]^{T} .
\end{aligned}
$$

Thus

$$
\mathcal{N}^{(k)}=(\mathbf{u}-\mathbf{w}-\mathbf{A} \mathbf{v})^{*}(\mathbf{u}-\mathbf{w}-\mathbf{A} \mathbf{v}) .
$$

Here, the operator ${ }^{T}$ is used for the regular transposition, while ${ }^{*}$ applied to matrices and vectors is the Hermite transposition. For any $c, \mathcal{N}^{(k)}$ attains its minimum $\mathcal{N}_{m}^{(k)}$ when $\mathbf{v}$ is the least square solution of equation $\mathbf{u}-\mathbf{w}-\mathbf{A} \mathbf{v}=0$, i.e.,

$$
\mathbf{v}=\mathbf{A}^{+}(\mathbf{u}-\mathbf{w}),
$$

where $\mathbf{A}^{+}=\left(A^{*} A\right)^{-1} A^{*}$ is the Penrose inverse of $\mathbf{A}[7]$. Consequently

$$
\mathcal{N}_{m}^{(k)}=(\mathbf{u}-\mathbf{w})^{*}\left(\mathbf{I}-\mathbf{A} \mathbf{A}^{+}\right)(\mathbf{u}-\mathbf{w}),
$$

Note that $\mathcal{N}_{m}^{(k)}$ depends on $c$ only. The problem becomes finding $c$ such that $\mathcal{N}_{m}^{(k)}$ is minimized.

In [8], we have derived a suitable expression for $\mathcal{N}_{m}^{(k)}$ which allows us to factorize its first derivative easily, and only one nontrivial factor yields the local minimum. Some recursive relations between the polynomials determining the multiple zeros for consecutive $k$ 's are also provided. 
In present paper we shall be concerned with the design of a practical algorithm. Furthermore, we include some new results that have not been published before. There is a sharp difference between the method by Karmarkar and Lakshman [4] [5] and the method in this paper: the former searches for the minimum point by an iterative numerical method, while the latter determines the point definitely by solving an equation.

The remaining part of the paper is set up as follows. First, we recall the approach and results in [8]. In section 3, we derive a new expression for the minimum of $\mathcal{N}_{m}^{(k)}$ which is easily computed and induces in particular, explicit expressions of the nearest singular polynomials for $k=2,3,4$. In the final section, we estimate the precision required in the computation of the multiple root and describe the algorithm for computing the nearest singular polynomial, multiple root and the minimal perturbation. The performance of the algorithm is illustrated by several numerical examples.

\section{Preliminaries}

In order to obtain explicit expression for $\mathcal{N}_{m}^{(k)}$ and the nearest singular polynomial, let us introduce polynomial

$$
q_{1}:=\sum_{j=0}^{m-1}(c \bar{c})^{j},
$$

the vector

$$
\mathbf{e}_{k}:=\left[f(c), f^{\prime}(c), \ldots, f^{(k-1)}(c)\right]^{T}
$$

and matrix $\boldsymbol{\Lambda}_{k}$

$$
\boldsymbol{\Lambda}_{k}:=\left[\begin{array}{cccc}
q_{1} & \frac{\partial q_{1}}{\partial \bar{c}} & \cdots & \frac{\partial^{k-1} q_{1}}{\partial^{k-1}} \\
\frac{\partial q_{1}}{\partial c} & \frac{\partial^{2} q_{1}}{\partial \partial \partial \bar{c}} & \cdots & \frac{\partial^{k} q_{1}}{\partial c \partial^{k-1} \bar{c}} \\
\vdots & \vdots & \ddots & \vdots \\
\frac{\partial^{k-1} q_{1}}{\partial^{k-1} c} & \frac{\partial^{k} q_{1}}{\partial^{k-1} c \partial \bar{c}} & \cdots & \frac{\partial^{2 k-2} q_{1}}{\partial^{k-1} c \partial^{k-1} \bar{c}}
\end{array}\right] \in \mathbb{C}^{k \times k}
$$

Theorem $1 \Lambda_{k}$ is a Hermitian and positive definite matrix, and

$$
\operatorname{det}\left(\boldsymbol{\Lambda}_{k}\right) \geq\left(\prod_{i=1}^{k}(k-i) !\right)^{2}
$$

Proof. The matrix $\boldsymbol{\Lambda}_{k}$ is factorized as

$$
\Lambda_{k}=J \Omega^{-1} V V^{*}\left(\Omega^{-1}\right)^{*} J,
$$

where 


$$
\begin{aligned}
& J=\left[\begin{array}{ccccc}
0 & \ldots & 0 & 0 & 1 \\
0 & \ldots & 0 & 1 & 0 \\
0 & \ldots & 1 & 0 & 0 \\
\vdots & \ddots & \vdots & \vdots & \vdots \\
1 & \ldots & 0 & 0 & 0
\end{array}\right]_{k \times k} \\
& \Omega=\left[\begin{array}{ccccc}
\frac{1}{(k-1) !} & 0 & 0 & \ldots & 0 \\
\frac{1}{(k-1) !} c & \frac{1}{(k-2) !} & 0 & \ldots & 0 \\
\frac{1}{(k-1) !} c^{2} & \frac{2}{(k-2) !} c & \frac{1}{(k-3) !} & \ldots & 0 \\
\vdots & \vdots & \vdots & \ddots & \vdots \\
\frac{1}{(k-1) !} c^{k-1} & \frac{k-1}{(k-2) !} c^{k-2} & \frac{(k-1)}{(k-3) !} c^{k-3} & \ldots & 1
\end{array}\right]_{k \times k}
\end{aligned}
$$

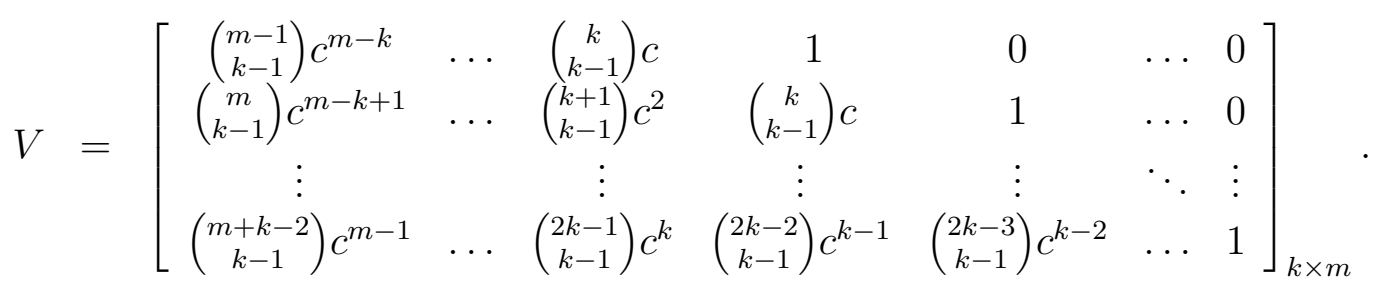

where $\Omega_{i j}=\frac{1}{(k-j) !}\left(\begin{array}{c}i-1 \\ j-1\end{array}\right) c^{i-j}$ and $V_{i j}=\left(\begin{array}{c}m+i-j-1 \\ k-1\end{array}\right) c^{m-k+i-j}$.

We split the matrix $V=\left[\begin{array}{ll}V_{1} & V_{2}\end{array}\right]$, into the first $m-k$ and the last $k$ columns, then it is straightforward to see that

$$
V V^{*}=\left[\begin{array}{ll}
V_{1} & V_{2}
\end{array}\right]\left[\begin{array}{c}
V_{1}^{*} \\
V_{2}^{*}
\end{array}\right]=V_{1} V_{1}^{*}+V_{2} V_{2}^{*}
$$

Since the symmetric matrix $V_{2} V_{2}^{*}$ is a positive definite matrix and the symmetric matrix $V_{1} V_{1}^{*}$ is a semidefinite matrix, then the symmetric matrix $V V^{*}=V_{1} V_{1}^{*}+$ $V_{2} V_{2}^{*}$ is a positive definite matrix and the product of its eigenvalues is bigger than the product of all eigenvalues of $V_{2} V_{2}^{*}$ [1]. We know the product of all eigenvalues of $V_{2} V_{2}^{*}$ is equal to its determinant which is 1 . So we have $\operatorname{det}\left(V V^{*}\right) \geq 1$. It follows that

$$
\operatorname{det}\left(\boldsymbol{\Lambda}_{k}\right)=\operatorname{det}(J) \operatorname{det}\left(\Omega^{-1}\right) \operatorname{det}\left(V V^{*}\right) \operatorname{det}\left(\left(\Omega^{-1}\right)^{*}\right) \operatorname{det}(J) \geq\left(\prod_{i=1}^{k}(k-i) !\right)^{2}
$$

We now consider the expression of the $\mathcal{N}_{m}^{(k)}$ and its derivative. For reference, we restate the following three theorems in [8]. 
Theorem 2 The $\mathcal{N}_{m}^{(k)}$ and its derivative can be expressed as:

$$
\begin{gathered}
\mathcal{N}_{m}^{(k)}=\mathbf{e}_{k}^{*} \boldsymbol{\Lambda}_{k}^{-1} \mathbf{e}_{k} \\
\frac{\partial \mathcal{N}_{m}^{(k)}}{\partial c}=\frac{p_{k+1} \overline{p_{k}}}{q_{k}^{2}}, \quad q_{k}:=\operatorname{det} \boldsymbol{\Lambda}_{k}, \quad p_{k}:=\operatorname{det} \mathbf{P}_{k},
\end{gathered}
$$

where

$$
\mathbf{P}_{k}:=\left[\begin{array}{cccc}
q_{1} & \cdots & \frac{\partial^{k-2} q_{1}}{\partial q^{k-2}} & f(c) \\
\frac{\partial q_{1}}{\partial c} & \cdots & \frac{\partial^{k-1} q_{1}}{\partial c \partial^{k-2} \bar{c}} & f^{\prime}(c) \\
\vdots & \ddots & \vdots & \vdots \\
\frac{\partial^{k-1} q_{1}}{\partial^{k-1} c} & \cdots & \frac{\partial^{2 k-3} q_{1}}{\partial^{k-1} c \partial^{k-2} \bar{c}} & f^{(k-1)}(c)
\end{array}\right] \in \mathbb{C}^{k \times k}
$$

Theorem $3 \mathcal{N}_{m}^{(k)}$ attains its local minimum only if $c$ satisfies $p_{k}=0$ and

$$
q_{k-1}^{4} p_{k+1} \overline{p_{k+1}}-q_{k}^{4} p_{k-1} \overline{p_{k-1}} \geq 0 .
$$

The following theorem gives an alternative method to determine $p_{i}$ and $q_{i}$ in (7) (9) for $i=k-1, k, k+1$.

Theorem 4 Let $q_{0}:=1, q_{1}:=\sum_{j=0}^{m-1}(c \bar{c})^{j}$ and $p_{1}:=f(c), q_{i}, p_{i}$ for $1<i \leq k+1$ can be computed by the following recursive relations:

$$
\begin{aligned}
q_{i} \frac{\partial^{2} q_{i}}{\partial c \partial \bar{c}}-\frac{\partial q_{i}}{\partial c} \frac{\partial q_{i}}{\partial \bar{c}} & =q_{i-1} q_{i+1} \\
p_{i} \frac{\partial^{2} p_{i}}{\partial c \partial \bar{c}}-\frac{\partial p_{i}}{\partial c} \frac{\partial p_{i}}{\partial \bar{c}} & =p_{i-1} p_{i+1} \\
q_{i} \frac{\partial p_{i}}{\partial c}-p_{i} \frac{\partial q_{i}}{\partial c} & =q_{i-1} p_{i+1} .
\end{aligned}
$$

Notice that if the coefficients of $f(x)$ are floating points, we will first consider these coefficients as symbols and substitute them after exact computation of the $p_{i}$ and $q_{i}$. In this way, if the expressions of $p_{i}$ and $q_{i}$ are computed once, they can also be used for other polynomials of the same degree but different coefficients as $f(x)$. The formulas (10)(12) are used in the Algorithm $\mathrm{N}$ to compute $q_{i}$ and $p_{i}$ recursively.

\section{Another Expression for $\mathcal{N}_{m}^{(k)}$}

The expression (6) of $\mathcal{N}_{m}^{(k)}$ is very suitable for deducing the simple formula of its derivative (7), but the inverse of matrix in (6) makes it uneasy to calculate for large $k$. Here we provide an alternative explicit expression for $\mathcal{N}_{m}^{(k)}$ in terms of $q_{i}$ and $p_{i}$. 


\section{Theorem 5}

$$
\mathcal{N}_{m}^{(k)}=\frac{p_{1} \overline{p_{1}}}{q_{1}}+\frac{p_{2} \overline{p_{2}}}{q_{1} q_{2}}+\cdots+\frac{p_{k} \overline{p_{k}}}{q_{k-1} q_{k}} .
$$

Proof. For $k=1$, it is obvious since $\boldsymbol{\Lambda}_{1}=\left[q_{1}\right]$ and $\mathbf{e}_{1}=[f(c)]=\left[p_{1}\right]$. Suppose equation (13) is true for $1 \leq i \leq k$. We partition the matrix $\boldsymbol{\Lambda}_{k+1}$ as

$$
\boldsymbol{\Lambda}_{k+1}=\left[\begin{array}{cc}
\boldsymbol{\Lambda}_{k} & \mathbf{w}_{k} \\
\mathbf{w}_{k}^{*} & \alpha
\end{array}\right]
$$

where

$$
\mathbf{w}_{k}=\left[\frac{\partial^{k} q_{1}}{\partial^{k} \bar{c}}, \frac{\partial^{k+1} q_{1}}{\partial c \partial^{k} \bar{c}}, \ldots, \frac{\partial^{2 k-1} q_{1}}{\partial^{k-1} c \partial^{k} \bar{c}}\right]^{T}, \quad \alpha=\frac{\partial^{2 k} q_{1}}{\partial^{k} c \partial^{k} \bar{c}} .
$$

Since the matrix $\boldsymbol{\Lambda}_{k}$ is nonsingular by Theorem 1, applying the Gaussian elimination, we obtain

$$
\left[\begin{array}{cc}
\mathbf{I} & \mathbf{0} \\
-\mathbf{w}_{k}^{*} \boldsymbol{\Lambda}_{k}^{-1} & 1
\end{array}\right]\left[\begin{array}{cc}
\boldsymbol{\Lambda}_{k} & \mathbf{w}_{k} \\
\mathbf{w}_{k}^{*} & \alpha
\end{array}\right]=\left[\begin{array}{cc}
\boldsymbol{\Lambda}_{k} & \mathbf{w}_{k} \\
\mathbf{0} & \alpha-\mathbf{w}_{k}^{*} \boldsymbol{\Lambda}_{k}^{-1} \mathbf{w}_{k}
\end{array}\right]
$$

Compare the determinant of the matrices on the two sides, we have

$$
\beta:=\alpha-\mathbf{w}_{k}^{*} \boldsymbol{\Lambda}_{k}^{-1} \mathbf{w}_{k}=\frac{\operatorname{det} \boldsymbol{\Lambda}_{k+1}}{\operatorname{det} \boldsymbol{\Lambda}_{k}}=\frac{q_{k+1}}{q_{k}} .
$$

Now the inverse of $\boldsymbol{\Lambda}_{k+1}$ can be expressed through the inverse of $\boldsymbol{\Lambda}_{k}$ :

$$
\boldsymbol{\Lambda}_{k+1}^{-1}=\left[\begin{array}{cc}
\boldsymbol{\Lambda}_{k}^{-1} & -\boldsymbol{\Lambda}_{k}^{-1} \mathbf{w}_{k} \beta^{-1} \\
\mathbf{0} & \beta^{-1}
\end{array}\right]\left[\begin{array}{cc}
\mathbf{I} & \mathbf{0} \\
-\mathbf{w}_{k}^{*} \boldsymbol{\Lambda}_{k}^{-1} & 1
\end{array}\right]
$$

By inserting the expression of $\boldsymbol{\Lambda}_{k+1}^{-1}$ into (6) for $k+1$, we obtain

$$
\begin{aligned}
\mathcal{N}_{m}^{(k+1)} & =\left[\mathbf{e}_{k}^{*}, \overline{f^{(k)}(c)}\right]\left[\begin{array}{cc}
\boldsymbol{\Lambda}_{k}^{-1} & -\boldsymbol{\Lambda}_{k}^{-1} \mathbf{w}_{k} \beta^{-1} \\
\mathbf{0} & \beta^{-1}
\end{array}\right]\left[\begin{array}{cc}
\mathbf{I} & \mathbf{0} \\
-\mathbf{w}_{k}^{*} \boldsymbol{\Lambda}_{k}^{-1} & 1
\end{array}\right]\left[\begin{array}{c}
\mathbf{e}_{k} \\
f^{(k)}(c)
\end{array}\right] \\
& =\mathbf{e}_{k}^{*} \boldsymbol{\Lambda}_{k}^{-1} \mathbf{e}_{k}+\beta^{-1}\left(\overline{f^{(k)}(c)}-\mathbf{e}_{k}^{*} \boldsymbol{\Lambda}_{k}^{-1} \mathbf{w}_{k}\right)\left(f^{(k)}(c)-\mathbf{w}_{k}^{*} \boldsymbol{\Lambda}_{k}^{-1} \mathbf{e}_{k}\right) \\
& =\mathcal{N}_{m}^{(k)}+\frac{q_{k}}{q_{k+1}} \frac{\overline{p_{k+1}}}{\frac{q_{k}}{p_{k+1}}} \\
& =\mathcal{N}_{m}^{(k)}+\frac{p_{k+1}}{q_{k} q_{k+1}}
\end{aligned}
$$

From the expression (13), it is clear that the minimum $\mathcal{N}_{m}^{(k)}$ increases along with the multiplicity $k$. According to Theorem $2, \mathcal{N}_{m}^{(k)}$ attains its local minimum only if the value of $c$ satisfies $p_{k}=0$. So (13) can be simplified to

$$
\mathcal{N}_{m}^{(k)}=\frac{p_{1} \overline{p_{1}}}{q_{1}}+\frac{p_{2} \overline{p_{2}}}{q_{1} q_{2}}+\cdots+\frac{p_{k-1} \overline{p_{k-1}}}{q_{k-2} q_{k-1}} .
$$


By now, we have expressed $\mathcal{N}_{m}^{(k)}$ and $\partial \mathcal{N}_{m}^{(k)} / \partial c$ explicitly in terms of $p_{i}$ and $q_{i}$ which can be calculated recursively. In the sequel we also try to set up some formulas based on $q_{i}$ instead of (2) to compute the coefficients of the corresponding nearest singular polynomials.

For $k=2$, suppose $c=\gamma$ gives the minimum of $\mathcal{N}_{m}^{(2)}$. Let

$$
h_{2}(x):=f(x)-\frac{f(\gamma)}{q_{1}} q_{11}(x),
$$

where

$$
q_{11}(x):=\sum_{i=0}^{m-1}(\bar{c} x)^{i} .
$$

It is trivial to check that $h_{2}(\gamma)=0$ and $h_{2}^{\prime}(\gamma)=p_{2} / q_{1}=0$. Thus $h_{2}(x)$ has a double root $\gamma$ and

$$
\left\|f-h_{2}\right\|^{2}=\frac{f(\gamma) \overline{f(\gamma)}}{q_{1}} \leq \mathcal{N}_{m}^{(2)} .
$$

So $h_{2}$ is the nearest singular polynomial of $f$ with a root of multiplicity 2 . Similar results can be found in [5] [2] [6].

It turns out that analogous formulas exist for $k=3,4$.

Theorem 6 For $k=3$, suppose $c=\gamma$ gives the minimum of $\mathcal{N}_{m}^{(3)}$, then the nearest singular polynomial is

$$
h_{3}(x):=h_{2}(x)-\frac{h_{2}^{\prime}(\gamma)}{q_{2}} q_{22}(x),
$$

where $h_{2}(x)$ is defined as (15) and

$$
q_{22}(x):=q_{1} \frac{\partial q_{11}(x)}{\partial \bar{c}}-\frac{\partial q_{1}}{\partial \bar{c}} q_{11}(x) .
$$

Proof. It is easy to check that $q_{22}(\gamma)=0, q_{22}^{\prime}(\gamma)=q_{2}$ and $q_{22}^{\prime \prime}(\gamma)=\partial q_{2} / \partial c$. Therefore, $h_{3}(\gamma)=h_{3}^{\prime}(\gamma)=0$ and

$$
\begin{array}{rlr}
h_{3}^{\prime \prime}(\gamma) & =\frac{1}{q_{1}} \frac{\partial p_{2}}{\partial c}-\frac{p_{2}}{q_{1} q_{2}} \frac{\partial q_{2}}{\partial c} \\
& =\frac{q_{1} p_{3}}{q_{1} q_{2}} \quad \text { (by Theorem 4) } \\
& =0 . \quad \text { (by Theorem 3) }
\end{array}
$$

It follows that $\gamma$ is a triple root of $h_{3}(x)$. Since

$$
\begin{aligned}
\left\|q_{22}\right\|^{2} & =\sum_{i=0}^{m-1}\left(i q_{1} \bar{c}^{i-1}-\frac{\partial q_{1}}{\partial \bar{c}} \bar{c}^{i}\right)\left(i q_{1} c^{i-1}-\frac{\partial q_{1}}{\partial c} c^{i}\right) \\
& =q_{1}\left(q_{1} \frac{\partial^{2} q_{1}}{\partial c \partial \bar{c}}-\frac{\partial q_{1}}{\partial c} \frac{\partial q_{1}}{\partial \bar{c}}\right) \\
& =q_{1} q_{2},
\end{aligned}
$$


we have

$$
\begin{aligned}
\left\|h_{3}-f\right\|^{2} & \leq\left\|h_{3}-h_{2}\right\|^{2}+\left\|h_{2}-f\right\|^{2} \\
& \leq \frac{p_{2} \bar{p}_{2}}{q_{1}^{2} q_{2}^{2}}\left\|q_{22}\right\|^{2}+\frac{f(\gamma) \overline{f(\gamma)}}{q_{1}}=\mathcal{N}_{m}^{(3)}
\end{aligned}
$$

Theorem 7 For $k=4$, suppose $c=\gamma$ gives the minimum of $\mathcal{N}_{m}^{(4)}$, then the nearest singular polynomial is

$$
h_{4}(x):=h_{3}(x)-\frac{h_{3}^{\prime \prime}(\gamma)}{q_{3}} q_{33}(x),
$$

where

$$
q_{33}(x):=\frac{1}{q_{1}}\left(q_{2} \frac{\partial q_{22}(x)}{\partial \bar{c}}-\frac{\partial q_{2}}{\partial \bar{c}} q_{22}(x)\right) .
$$

Proof From the proof of the above theorems, we know that $h_{4}(\gamma)=0$ and $q_{33}(\gamma)=$ $0, q_{33}^{\prime}(\gamma)=0, q_{33}^{\prime \prime}(\gamma)=q_{3}, q_{33}^{(3)}(\gamma)=\frac{\partial q_{3}}{\partial c}$. So, $h_{4}(\gamma)=h_{4}^{\prime}(\gamma)=h_{4}^{\prime \prime}(\gamma)=0$ and

$$
\begin{aligned}
h_{4}^{(3)}(\gamma) & =\frac{1}{q_{2}} \frac{\partial p_{3}}{\partial c}-\frac{p_{3}}{q_{2} q_{3}} \frac{\partial q_{3}}{\partial c} \\
& =\frac{q_{2} p_{4}}{q_{2} q_{3}}=0 .
\end{aligned}
$$

It follows that $\gamma$ is a root of $h_{3}(x)$ of multiplicity 4 . Since

$$
\begin{aligned}
\left\|q_{1} q_{33}\right\|^{2}= & \sum_{i=0}^{m-1}\left(q_{2}\left(i(i-1) q_{1} \bar{c}^{i-2}-\frac{\partial^{2} q_{1}}{\partial \bar{c}^{2}} \bar{c}^{i}\right)-\frac{\partial q_{2}}{\partial \bar{c}}\left(i q_{1} \bar{c}^{i-1}-\frac{\partial q_{1}}{\partial \bar{c}} \bar{c}^{i}\right)\right) \\
& \left(q_{2}\left(i(i-1) q_{1} c^{i-2}-\frac{\partial^{2} q_{1}}{\partial c^{2}} c^{i}\right)-\frac{\partial q_{2}}{\partial c}\left(i q_{1} c^{i-1}-\frac{\partial q_{1}}{\partial c} c^{i}\right)\right) \\
= & q_{1}^{2} q_{2} q_{3},
\end{aligned}
$$

we have

$$
\begin{aligned}
\left\|h_{4}-f\right\|^{2} & \leq\left\|h_{4}-h_{3}\right\|^{2}+\left\|h_{3}-f\right\|^{2} \\
& \leq \frac{p_{3} \bar{p}_{3}}{q_{2}^{2} q_{3}^{2}}\left\|q_{33}\right\|^{2}+\frac{p_{2} \bar{p}_{2}}{q_{1} q_{2}}+\frac{p_{1} \bar{p}_{1}}{q_{1}}=\mathcal{N}_{m}^{(4)} .
\end{aligned}
$$

Unfortunately, there are no obvious way to generalize $(15)(16)(17)$ to the cases of $k>4$. The study in this point is in progress and will be reported in the future. 


\section{Algorithm and Examples}

Let $\gamma=a+\mathbf{i} b$, where $a$ and $b$ are real, $\mathbf{i}$ stands for the imaginary unit. By Theorem 3 , we subject to find the real solutions of a polynomial equations system. It consists of the real and imaginary part of $p_{k}(a, b)$. In order to guarantee the absolute minimum of $\mathcal{N}_{m}^{(k)}$ to be within $\delta^{2}$, we estimate the precision required in the calculation of real root $a$ and $b$ of $p_{k}(a, b)$. The analysis is similar to [5], but it is much simple and clear due to the explicit expressions we have derived. Since $\gamma=a+\mathbf{i} b$ is a multiple root of the minimally perturbed polynomial $h$ whose coefficients are bounded in magnitude by the coefficients of $f$. Therefore we suppose $\|a+\mathbf{i} b\| \leq B$, where $B=1+2\|f\|$.

Theorem 8 For a given $\delta>0, \gamma=a+\mathbf{i} b$, if

$$
\delta_{a}, \delta_{b}<\delta m^{-2(k+1)^{2}} B^{-2 m(k+1)}
$$

then $\mathcal{N}_{m}^{(k)}\left(a+\delta_{a}, b+\delta_{b}\right)-\mathcal{N}_{m}^{(k)}(a, b) \leq \delta^{2}$

Proof. We have

$$
\mathcal{N}_{m}^{(k)}\left(a+\delta_{a}, b+\delta_{b}\right)-\mathcal{N}_{m}^{(k)}(a, b) \leq \frac{\partial \mathcal{N}_{m}^{(k)}(a, b)}{\partial c} \delta_{a} \delta_{b} \leq \frac{p_{k+1} \overline{p_{k}}}{q_{k}^{2}} \delta_{a} \delta_{b}
$$

By Theorem $1, q_{k} \geq\left(\prod_{i=1}^{k}(k-i) !\right)^{2} \geq 1$. It follows that only bounds of $p_{k}(a, b), p_{k+1}(a, b)$ are needed. Using the recursive relations in Theorem 4 , we get

$$
\operatorname{deg}_{\gamma} p_{k+1}+\operatorname{deg}_{\gamma} p_{k-1} \leq 2 \operatorname{deg}_{\gamma} p_{k} .
$$

Therefore,

$$
\operatorname{deg}_{\gamma} p_{k} \leq k \operatorname{deg}_{\gamma} p_{1}=m k .
$$

Similarly it can be shown that

$$
\operatorname{deg}_{\bar{\gamma}} p_{k} \leq(m-1) k
$$

The bound of coefficients in $p_{k}$ can be obtained directly from the estimate of entries in matrix (8) as $m^{2 k^{2}} B$. Note that we have assumed $\|a+\mathbf{i} b\| \leq B$. Thus,

$\mathcal{N}_{m}^{(k)}\left(a+\delta_{a}, b+\delta_{b}\right)-\mathcal{N}_{m}^{(k)}(a, b) \leq \delta_{a} \delta_{b} m^{2 k^{2}} B^{2 m k} m^{2(k+1)^{2}} B^{2 m(k+1)} \leq \delta_{a} \delta_{b} m^{4(k+1)^{2}} B^{4 m(k+1)}$.

The (18) implies that

$$
\mathcal{N}_{m}^{(k)}\left(a+\delta_{a}, b+\delta_{b}\right)-\mathcal{N}_{m}^{(k)}(a, b) \leq \delta^{2}
$$

We are now ready to describe the algorithm for computing the nearest singular polynomials with a $k$-fold root. 


\section{Algorithm N:}

Input: Monic polynomial $f \in \mathbb{C}[x]$ of degree $m$, an error bound $\delta$ and an integer $k>1$.

Output: The nearest singular polynomial $h$ and $\gamma=a+\mathbf{i} b \in \mathbb{C}$ such that $\gamma$ is the $k$-fold root of $h$ and $\mathcal{N}_{m}^{(k)}(a, b)-\left(\right.$ absolute minimum of $\left.\mathcal{N}_{m}^{(k)}\right) \leq \delta^{2}$.

N1. Set $q_{0}:=1, q_{1}:=\sum_{j=0}^{m-1}(c \bar{c})^{j}$ and $p_{1}:=f(c)$.

N2. For $i$ from 2 to $k+1$ do

$$
\begin{aligned}
q_{i} & :=\frac{1}{q_{i-2}}\left(q_{i-1} \frac{\partial^{2} q_{i-1}}{\partial c \partial \bar{c}}-\frac{\partial q_{i-1}}{\partial c} \frac{\partial q_{i-1}}{\partial \bar{c}}\right) \text { by formula (10); } \\
p_{i} & :=\frac{1}{q_{i-2}}\left(q_{i-1} \frac{\partial p_{i-1}}{\partial c}-p_{i-1} \frac{\partial q_{i-1}}{\partial c}\right) \text { by formula (12). }
\end{aligned}
$$

N3. Find the roots of $p_{k}$ satisfying inequality (9) and bounded by $B$ to the precision defined in Theorem 8.

N4. Compute $\mathcal{N}_{m}^{(k)}$ by the formula (14). Let $\gamma=a+\mathbf{i} b$ be one of the roots giving the least value of $\mathcal{N}_{m}^{(k)}$.

N5. For $k=2,3,4$, determine the nearest singular polynomial $h$ by formulas (15) (16) (17) respectively. Otherwise $h$ is obtained from equation (2).

The algorithm has been implemented in the computer algebra system Maple $\mathrm{V}$ Release 5. Numerical experiments were run on a DEC personal workstation $(433 \mathrm{MHz}$, 256Mbyte). The following table presents times(seconds) for running Algorithm $\mathrm{N}$ on five examples (see Appendix). The times are broken down into symbolic deduction of the expressions of $p_{k}, h_{k}, \mathcal{N}_{m}^{(k)}$ depending on $c, \bar{c}$, numerically finding the multiple root $\gamma$, and evaluating $h_{k}, \mathcal{N}_{m}^{(k)}$ at $\gamma$. Ex and $k$ show the example number and multiplicity respectively. For the details of the computation, see the Appendix.

\begin{tabular}{|c|c|c|c|c|}
\hline Ex & $k$ & $\begin{array}{c}\text { Symbolic deduction } \\
\text { time }(\mathrm{s})\end{array}$ & $\begin{array}{c}\text { Finding root } \\
\text { time }(\mathrm{s})\end{array}$ & $\begin{array}{c}\text { Evaluation } \\
\text { time }(\mathrm{s})\end{array}$ \\
\hline 1 & 2 & 0.02 & 0.02 & 0.01 \\
1 & 3 & 0.02 & 0.03 & 0.02 \\
1 & 4 & 0.02 & 0.02 & 0.03 \\
2 & 2 & 0.03 & 0.03 & 0.02 \\
3 & 2 & 0.09 & 0.03 & 0.01 \\
4 & 2 & 0.05 & 0.11 & 0.02 \\
4 & 3 & 0.11 & 0.05 & 0.02 \\
4 & 4 & 0.20 & 0.05 & 0.06 \\
5 & 2 & 0.03 & 1.17 & 0.02 \\
6 & 2 & 0.08 & 2.44 & 0.03 \\
6 & 3 & 0.20 & 18.05 & 0.15 \\
\hline
\end{tabular}




\section{Conclusion}

In this paper, we show how to compute the nearest singular polynomials by the technique of parametric minimization. The algorithm presented here is of polynomialtime complexity in the degree and size of the coefficients of the input polynomial. Our method has been applied to sensitivity analysis of root locations of polynomials subject to coefficient perturbations. However, for $k>4$, we have not yet obtained an explicit formula for the nearest singular polynomial with a given multiple zero. In fact, the algorithm appears to reach its limit for $k>4$. The practicality of our algorithm has still to be tested.

Acknowledgment The authors thank Prof.H.J.Stetter for many useful suggestions and the anonymous referees for their fruitful comments.

\section{Appendix. A Collection of Examples}

Let $k, h_{k}, \gamma$ and $\mathcal{N}_{m}^{(k)}$ be the same as above. For a given tolerance $\epsilon$, if $\mathcal{N}_{m}^{(k)} \leq \epsilon^{2}$, then we say $f$ has a $k$-cluster of zeros and $\gamma$ is called the center of the cluster [3]. In the following text, all coefficients are displayed to 7-decimals floating-point approximation.

\section{Example 1}

$$
f=x^{4}-0.960000 x^{3}-0.0401000 x^{2}+0.000096 x+0.000004 .
$$

For $k=2$ :

$$
\begin{aligned}
h_{2} & =x^{4}-0.960000 x^{3}-0.0401000 x^{2}+0.0000959953 x-0.0000000559041 \\
\gamma & =0.00114957 ; \quad(\text { double root }) \\
\mathcal{N}_{m}^{(2)} & =1.645038 \cdot 10^{-11}
\end{aligned}
$$

For $k=3$ :

$$
\begin{aligned}
h_{3} & =x^{4}-0.960000 x^{3}-0.0400826 x^{2}+0.000547512 x+0.00000248153 ; \\
\gamma & =-0.0135359 ; \quad(\text { triple root }) \\
\mathcal{N}_{m}^{(3)} & =4.144531 \cdot 10^{-7} .
\end{aligned}
$$

For $k=4$ :

$$
\begin{aligned}
h_{4} & =x^{4}-0.792494 x^{3}+0.235518 x^{2}-0.0311077 x+0.00154079 \\
\gamma & =0.198123 ; \quad \text { (quadruple root) } \\
\mathcal{N}_{m}^{(4)} & =0.104999
\end{aligned}
$$

Suppose the given accuracy $\epsilon=10^{-3}$, then $f$ has a 3-cluster of zeros . If $\epsilon=10^{-5}$, then $f$ only has a 2 -cluster of zeros. 


\section{Example 2}

$$
f=x^{5}+2.03 x^{4}-0.9398 x^{3}-2.0296 x^{2}-0.0602 x-0.0004 .
$$

For $k=2$ :

$$
\begin{aligned}
h_{2} & =x^{5}+2.030000 x^{4}-0.939800 x^{3}-2.029600 x^{2}-0.0601992 x-0.000449603 ; \\
\gamma & =-0.0149931 ; \quad(\text { double root }) \\
\mathcal{N}_{m}^{(2)} & =2.460988 \cdot 10^{-9} .
\end{aligned}
$$

Since $\mathcal{N}_{m}^{(2)}$ is very small, we try $k=3$ to see if there is a 3 -cluster of zeros. For $k=3, \mathcal{N}_{m}^{(3)}=0.368179$, so it is only possible for $f$ has a 2 -cluster.

\section{Example 3}

$$
f=x^{6}+1.99 x^{5}-9.0202 x^{4}-1.9104 x^{3}+8.0218 x^{2}-0.0796 x-0.0016 .
$$

For $k=2$ :

$$
\begin{aligned}
h_{2}= & x^{6}+1.990000 x^{5}-9.020200 x^{4}-1.910400 x^{3}+8.021800 x^{2}-0.0795911 x \\
& +0.000197662 ; \\
\gamma= & 0.00497002 ; \quad(\text { double root }) \\
\mathcal{N}_{m}^{(2)}= & 3.231668 \cdot 10^{-6} .
\end{aligned}
$$

For $k=3, \mathcal{N}_{m}^{(3)}=5.766062, f$ has no 3-cluster of zeros.

\section{Example 4}

$$
f=x^{6}+2.04 x^{5}-0.9199 x^{4}-2.039806 x^{3}-0.080112 x^{2}-0.000194 x+0.000012 .
$$

For $k=2$ :

$$
\begin{aligned}
h_{2}= & x^{6}+2.040000 x^{5}-0.919900 x^{4}-2.039806 x^{3}-0.0801120 x^{2}-0.000194044 x \\
& +0.0000137343 ; \\
\gamma= & -0.0253447 ; \quad(\text { double root }) \\
\mathcal{N}_{m}^{(2)}= & 3.009789 \cdot 10^{-12} .
\end{aligned}
$$

For $k=3$ :

$$
\begin{aligned}
h_{3}= & x^{6}+2.040000 x^{5}-0.919900 x^{4}-2.039806 x^{3}-0.0800891 x^{2}+0.00105689 x \\
& +0.00000465900 ; \\
\gamma= & -0.0132538 ; \quad \text { (triple root) } \\
\mathcal{N}_{m}^{(3)}= & 7.453849 \cdot 10^{-7} .
\end{aligned}
$$


For $k=4$ :

$$
\begin{aligned}
h_{4}= & x^{6}+2.095264 x^{5}-1.073789 x^{4}-1.684835 x^{3}-0.612729 x^{2}-0.0921448 x \\
& -0.00507403 ; \\
\gamma= & -0.214203 ; \quad \text { (quadruple root) } \\
\mathcal{N}_{m}^{(4)}= & 0.444902 .
\end{aligned}
$$

So it is reasonable that $f$ has a 3-cluster of zeros if $\epsilon=10^{-3}$.

\section{Example 5}

$f=x^{5}+(0.909091+0.1 \mathbf{i}) x^{4}-10 . x^{3}-(9.09091-\mathbf{i}) x^{2}+9 . x+8.181818+0.9 \mathbf{i}$.

For $k=2$ :

$$
\begin{aligned}
h_{2}= & x^{5}+(0.904823+0.0863028 \mathbf{i}) x^{4}+(-9.996349+0.0145469 \mathbf{i}) x^{3} \\
& +(-9.093866-1.015397 \mathbf{i}) x^{2}+(9.002183+0.0162447 \mathbf{i}) x \\
& +(8.180493+0.882916 \mathbf{i}) ; \\
\gamma= & -0.955062-0.0536859 \mathbf{i} ; \quad \text { (double root) } \\
\mathcal{N}_{m}^{(2)}= & 0.00123884 .
\end{aligned}
$$

Suppose the given accuracy $\epsilon=10^{-1}$, then $f$ has a 2-cluster of zeros. Otherwise $f$ only has simple roots.

\section{Example 6}

$$
\begin{aligned}
f= & x^{21}-1.142857 x^{20}-1.0 x^{19}+2.714286 x^{18}-4.0 x^{17}+4.1428714 x^{16} \\
& -2.571371 x^{15}+x^{14}+0.857143 x^{13}-3.142857 x^{12}+2.0 x^{11}+0.285714 x^{10} \\
& +0.571428 x^{8}-1.285600 x^{7}+2.857143 x^{6}-4.714286 x^{5}+2.142857 x^{4} \\
& +0.428571 x^{3}+0.857143 x^{2}-0.714286 x-0.285700 .
\end{aligned}
$$

For $k=2$ :

$$
\begin{aligned}
h_{2}= & x^{21}-1.142866 x^{20}-1.000009 x^{19}+2.714276 x^{18}-4.000009 x^{17}+4.142862 x^{16} \\
& -2.571380 x^{15}+1.0 x^{14}+0.857133 x^{13}-3.142867 x^{12}+2.0 x^{11}+0.285705 x^{10} \\
& -0.95239110^{-5} x^{9}+0.571419 x^{8}-1.285609 x^{7}+2.857133 x^{6}-4.714295 x^{5} \\
& +2.142847 x^{4}+0.428562 x^{3}+0.857133 x^{2}-0.714295 x-0.285709 ; \\
\gamma= & .999991 ; \quad \text { (double root) } \\
\mathcal{N}_{m}^{(2)}= & .19047710^{-8} .
\end{aligned}
$$


For $k=3$ :

$$
\begin{aligned}
h_{3}= & x^{21}-1.142414 x^{20}-0.999574 x^{19}+2.714695 x^{18}-3.999607 x^{17}+4.143250 x^{16} \\
& -2.571008 x^{15}+1.000349 x^{14}+0.857479 x^{13}-3.142534 x^{12}+2.000311 x^{11} \\
& +0.286013 x^{10}+0.00028743 x^{9}+0.571705 x^{8}-1.285334 x^{7}+2.857398 x^{6} \\
& -4.71404 x^{5}+2.143093 x^{4}+0.4287986 x^{3}+0.857361 x^{2}-0.714076 x \\
& -0.285498 ; \\
\gamma= & 1.040009 ; \quad \text { (triple root) } \\
\mathcal{N}_{m}^{(3)}= & 0.0000963776 .
\end{aligned}
$$

Suppose the given accuracy $\epsilon=10^{-2}$, then $f$ has a 3-cluster of zeros . If $\epsilon=10^{-4}$, then $f$ only has a 2-cluster of zeros.

\section{References}

[1] Gene H. Golub, Charles F, Van Loan Matrix Computations. John Hopkins U. Press, 3rd ed., 1996.

[2] Hitz, M.A., Kaltofen, E. Efficient algorithms for computing the nearest singular polynomials with constrained roots. In Proceedings of the 1998 International Symposium on Symbolic and Algebraic Computation, pp. 236-243.

[3] Hribernig, V., Stetter, H.J. Detection and validation of clusters of polynomial zeros. J. Symb. Comp.24(1997),667-681 (1997).

[4] Karmarkar, N., Lakshman Y.N. Approximate polynomial greatest common divisors and nearest singular polynomials. In Proceedings of the 1996 International Symposium on Symbolic and Algebraic Computation, pp. 35-39.

[5] Karmarkar, N., Lakshman, Y.N. On approximate polynomial greatest common divisors. J. Symb. Comp.26(6), 653-666 (1998).

[6] Manocha, D., Demmel, J. Algorithms for intersecting parametric and algebraic curves II: Multiple intersections. Computer Vision, Graphics and Image processing: Graphical Models and Image Processing 57, 2 (1995),pp. 81-100.

[7] C.R.Rao, S.K.Mitra Generalized Inverse of Matrices and its Applications. John Wiley 65 Sons, Inc, 1971.

[8] Zhi, L.H., Wu, W.D. Nearest singular polynomials. J. Symb. Comp.26(6) (1998), pp. 667-676. 